\title{
Stochastic fixed point theorems for a random Z-contraction in a complete probability measure space with application to non-linear stochastic integral equations
}

\author{
Plern Saiparaa ${ }^{\mathrm{a}}$, Poom Kumam ${ }^{\mathrm{a}, \mathrm{b}}$, Apirak Sombat ${ }^{\mathrm{a}}$, Anantachai Padcharoen ${ }^{\mathrm{a}}$, Wiyada Kumam ${ }^{\mathrm{c}, *}$ \\ ${ }^{a}$ KMUTTFixed Point Research Laboratory, Department of Mathematics, Room SCL 802 Fixed Point Laboratory, Science Laboratory \\ Building, Faculty of Science, King Mongkut's University of Technology Thonburi (KMUTT), 126 Pracha-Uthit Road, Bang Mod, Thrung \\ Khru, Bangkok 10140, Thailand. \\ ${ }^{b}$ KMUTT-Fixed Point Theory and Applications Research Group (KMUTT-FPTA), Theoretical and Computational Science Center (TaCS), \\ Science Laboratory Building, Faculty of Science, King Mongkut's University of Technology Thonburi (KMUTT), 126 Pracha-Uthit Road, \\ Bang Mod, Thrung Khru, Bangkok 10140, Thailand. \\ ${ }^{c}$ Program in Applied Statistics, Department of Mathematics and Computer Science, Faculty of Science and Technology, Rajamangala \\ University of Technology Thanyaburi, Thanyaburi (RMUTT), Pathumthani 12110, Thailand.
}

Communicated by 0 . Kolade Matthew

\begin{abstract}
In this paper, we propose the random z-contraction, prove a stochastic fixed point theorem for this contraction, and show that a solution for a non-linear stochastic integral equations exists in Banach spaces. (c)2017 All rights reserved.
\end{abstract}

Keywords: Z-contraction, stochastic fixed point theorem, complete probability measure spaces.

2010 MSC: 47H09, 47H10.

\section{Introduction}

The random fixed point theorems are stochastic generalizations of original fixed point theorems and need to use for the random equation theorems. In the same way the original fixed point theorems are the important of deterministic equation theorems. In 1955, Spacek [28] and Hans [7, 8] initiated to show the random fixed point theorems for some random contraction mappings in Polish spaces. In 1966, Mukherjee [19] provided a Schauder's random fixed point theorem over the space of atomic probability measure. In 1976, the work of Bharucha-Reid [5] allured the attention of various mathematic researchers and bring to the development of this theorem. In 1979, Itoh [9] extended theorems of Spacek and Hans to set-valued contraction mappings. Itoh [9] applied theorems of random fixed point to solve some type

\footnotetext{
${ }^{*}$ Corresponding author

Email addresses: plern.spn@mail.kmutt.ac.th (Plern Saipara), poom.kum@kmutt.ac.th (Poom Kumam), apirak.som@mail.kmutt.ac.th (Apirak Sombat), apadcharoen@yahoo.com (Anantachai Padcharoen), wiyada.kum@rmutt.ac.th (Wiyada Kumam)
}

doi:10.22436/mns.01.01.05 
of differential equations in Banach spaces. Sehgal et al. [27] obtained many theorems of random fixed point along with random analogue of the classical theorems based on work of Rothe [22]. The common random fixed points and random coincidence points of a pair of compatible random set-valued operators in Polish spaces were studied by Beg et al. [4]. Moreover, the concept of original random fixed point theorems became the source of inspiration for many new generation mathematics researchers working on the random fixed point theorems (for example, see in $[1,6,10,12-17,20,21,23-26]$ ).

In the sense of non-linear analysis, Banach's contraction principle [3] is very important to show a solution of some non-linear equations, differential and integral equations, and other non-linear problems exists. The following Banach's contraction principle, many authors have studied in several ways.

Theorem 1.1. If $(X, d)$ is a complete metric space and $\mathrm{T}: \mathrm{X} \rightarrow \mathrm{X}$ is a self-mapping so that

$$
d(T x, T y) \leqslant \alpha d(x, y)
$$

for each $x, y \in X$ and $\alpha \in[0,1)$, then $T$ has a unique fixed point.

Very recently, the new generalized Banach contraction is introduced by Khojasteh et al. [11] which they defined a simulation function and Z-contraction as follows.

Definition 1.2. Let $\zeta:[0, \infty) \times[0, \infty) \rightarrow \mathbb{R}$ be a mapping, then $\zeta$ is called a simulation function if it satisfies the assumptions as follows:

$\left(\Delta_{1}\right) \zeta(0,0)=0$;

$\left(\Delta_{2}\right) \zeta(t, s)<s-t$ for $t, s>0$;

$\left(\Delta_{3}\right)$ if $\left\{t_{n}\right\},\left\{s_{n}\right\}$ are sequences in $(0, \infty)$ so that

$$
\lim _{n \rightarrow \infty} t_{n}=\lim _{n \rightarrow \infty} s_{n}>0,
$$

then

$$
\limsup _{n \rightarrow \infty} \zeta\left(t_{n}, s_{n}\right)<0 .
$$

The set of all simulation functions is denoted by $z$.

Definition 1.3. Let $(X, d)$ be a metric space, $T$ is a self-mapping, and $\zeta \in Z$. Then $T$ is called a $Z$-contraction by respect to $\zeta$ if the following condition holds:

$$
\zeta(d(T x, T y), d(x, y)) \geqslant 0,
$$

where $x, y \in X$, with $x \neq y$.

Also, a non-linear stochastic analysis is an important mathematical discipline which is mostly connected with the study of some random operators. Their properties are important for the study of many random equations classes.

Since the importance of a non-linear stochastic analysis, we will propose the notion of random zcontraction and prove a stochastic fixed point theorem for this contraction operator. Furthermore, we apply our results for finding a solution for non-linear stochastic integral equations in Banach spaces.

\section{Stochastic fixed point results}

Motivated and inspired by Definition 1.3 and the work of Saha and Ganguly [26], we propose the definition of random $z$-contraction operators as follows.

Definition 2.1. Assume $T$ is a continuous random operator from $\Omega \times X$ to $X$. The operator $T$ is called random z-contraction if, for any $\omega \in \Omega$,

$$
\zeta\left(\left\|\mathrm{T}\left(\omega, x_{1}(\omega)\right)-\mathrm{T}\left(\omega, x_{2}(\omega)\right)\right\|,\left\|x_{1}(\omega)-x_{2}(\omega)\right\|\right) \geqslant 0
$$

for any random variables $x_{1}, x_{2}: \Omega \rightarrow X$. 
Now, we prove that a stochastic fixed point for random z-contraction exists in separable Banach spaces as follows.

Theorem 2.2. Assume that $(\Omega, \beta, \mu)$ is a complete probability measure space and $\mathrm{T}$ is an operator satisfying the random Z-contraction in Definition 2.1 almost surely for any $x_{1}(\omega), x_{2}(\omega) \in X$, where $X$ is a separable Banach space. Then a random fixed point of operator $T$ exists.

Proof. Suppose

$$
A=\{\omega \in \Omega: T(\omega, x) \text { is a continuous of } x\}
$$

and

$$
\mathrm{B}_{x_{1}, x_{2}}=\left\{\omega \in \Omega: \zeta\left(\left\|\mathrm{T}\left(\omega, x_{1}(\omega)\right)-\mathrm{T}\left(\omega, x_{2}(\omega)\right)\right\|,\left\|x_{1}(\omega)-x_{2}(\omega)\right\|\right) \geqslant 0\right\}
$$

Suppose $S$ is a set of countable dense, where $S \subset X$. Now, we prove that

$$
\bigcap_{x_{1}, x_{2} \in X}\left(B_{x_{1}, x_{2}} \cap A\right)=\bigcap_{s_{1}, s_{2} \in S}\left(B_{s_{1}, s_{2}} \cap A\right) \text {. }
$$

Then for all $s_{1}, s_{2} \in S$, we obtain

$$
\zeta\left(\left\|\mathrm{T}\left(\omega, \mathrm{s}_{1}(\omega)\right)-\mathrm{T}\left(\omega, \mathrm{s}_{2}(\omega)\right)\right\|,\left\|s_{1}(\omega)-s_{2}(\omega)\right\|\right) \geqslant 0,
$$

which implies that

$$
\left\|\mathrm{T}\left(\omega, s_{1}(\omega)\right)-\mathrm{T}\left(\omega, s_{2}(\omega)\right)\right\|<\left\|s_{1}(\omega)-s_{2}(\omega)\right\| .
$$

Because $S$ is dense subset of $X$, given by $\delta_{i}\left(x_{i}\right)>0$ there is $s_{1}, s_{2} \in S$ so that $\left\|x_{i}-s_{i}\right\|<\delta_{i}\left(x_{i}\right)$ for each $i=1,2$. Let $x_{1}, x_{2} \in X$. Now, we have

$$
\begin{aligned}
\left\|\mathrm{T}\left(\omega, x_{1}(\omega)\right)-\mathrm{T}\left(\omega, x_{2}(\omega)\right)\right\| \leqslant & \left\|\mathrm{T}\left(\omega, x_{1}(\omega)\right)-\mathrm{T}\left(\omega, \mathrm{s}_{1}(\omega)\right)\right\| \\
& +\left\|\mathrm{T}\left(\omega, \mathrm{s}_{1}(\omega)\right)-\mathrm{T}\left(\omega, \mathrm{s}_{2}(\omega)\right)\right\|+\left\|\mathrm{T}\left(\omega, \mathrm{s}_{2}(\omega)\right)-\mathrm{T}\left(\omega, \mathrm{x}_{2}(\omega)\right)\right\| .
\end{aligned}
$$

Substituting (2.1) in (2.2), we get

$$
\begin{aligned}
\left\|\mathrm{T}\left(\omega, x_{1}(\omega)\right)-\mathrm{T}\left(\omega, x_{2}(\omega)\right)\right\|< & \left\|\mathrm{T}\left(\omega, x_{1}(\omega)\right)-\mathrm{T}\left(\omega, s_{1}(\omega)\right)\right\| \\
& \left.+\left\|\mathrm{T}\left(\omega, s_{2}(\omega)\right)-\mathrm{T}\left(\omega, x_{2}(\omega)\right)\right\|\right)+\left\|s_{1}(\omega)-s_{2}(\omega)\right\| \\
\leqslant & \left.\left\|\mathrm{T}\left(\omega, x_{1}(\omega)\right)-\mathrm{T}\left(\omega, \mathrm{s}_{1}(\omega)\right)\right\|+\left\|\mathrm{T}\left(\omega, s_{2}(\omega)\right)-\mathrm{T}\left(\omega, x_{2}(\omega)\right)\right\|\right) \\
& +\left\|s_{1}(\omega)-x_{1}(\omega)\right\|+\left\|x_{1}(\omega)-x_{2}(\omega)\right\|+\left\|x_{2}(\omega)-s_{2}(\omega)\right\| .
\end{aligned}
$$

Because the function $T(\omega, x)$ is a continuous for all $\omega \in \Omega$, thus for all $\varepsilon>0$, there exists $\delta_{i}\left(x_{i}\right)>0$ for $i=1,2$ so that

$$
\left\|\mathrm{T}\left(\omega, x_{1}\right)-\mathrm{T}\left(\omega, \mathrm{s}_{1}\right)\right\|<\frac{\varepsilon}{8} \text { when }\left\|\mathrm{x}_{1}-\mathrm{s}_{1}\right\|<\delta_{1}\left(\mathrm{x}_{1}\right),
$$

and

$$
\left\|\mathrm{T}\left(\omega, x_{2}\right)-\mathrm{T}\left(\omega, \mathrm{s}_{2}\right)\right\|<\frac{\varepsilon}{8} \text { when }\left\|\mathrm{x}_{2}-\mathrm{s}_{2}\right\|<\delta_{1}\left(\mathrm{x}_{2}\right) .
$$

Now, we choose

$$
\delta_{1}=\min \left(\delta_{1}\left(x_{1}\right), \frac{\varepsilon}{8}\right)
$$

and

$$
\delta_{2}=\min \left(\delta_{2}\left(x_{2}\right), \frac{\varepsilon}{8}\right)
$$


For such a choice of $\delta_{1}, \delta_{2}$ by (2.3), we obtain

$$
\left\|\mathrm{T}\left(\omega, \mathrm{x}_{1}(\omega)\right)-\mathrm{T}\left(\omega, \mathrm{x}_{2}(\omega)\right)\right\|<\frac{\varepsilon}{8}+\frac{\varepsilon}{8}+\frac{\varepsilon}{8}+\left\|\mathrm{x}_{1}(\omega)-\mathrm{x}_{2}(\omega)\right\|+\frac{\varepsilon}{8}=\frac{\varepsilon}{2}+\left\|\mathrm{x}_{1}(\omega)-\mathrm{x}_{2}(\omega)\right\| .
$$

As $\varepsilon>0$ is arbitrary, if

$$
\left\|\mathrm{T}\left(\omega, x_{1}(\omega)\right)-\mathrm{T}\left(\omega, x_{2}(\omega)\right)\right\|<\left\|x_{1}(\omega)-x_{2}(\omega)\right\|,
$$

then

$$
\zeta\left(\left\|T\left(\omega, x_{1}(\omega)\right)-T\left(\omega, x_{2}(\omega)\right)\right\|,\left\|x_{1}(\omega)-x_{2}(\omega)\right\|\right) \geqslant 0 .
$$

Thus we have $\omega \in \bigcap_{x_{1}, x_{2} \in X}\left(B_{x_{1}, x_{2}} \cap A\right)$, which implies that

$$
\bigcap_{s_{1}, s_{2} \in S}\left(B_{s_{1}, s_{2}} \cap A\right) \subset \bigcap_{x_{1}, x_{2} \in X}\left(B_{x_{1}, x_{2}} \cap A\right)
$$

Also, we have

$$
\bigcap_{x_{1}, x_{2} \in X}\left(B_{x_{1}, x_{2}} \cap A\right) \subset \bigcap_{s_{1}, s_{2} \in S}\left(B_{s_{1}, s_{2}} \cap A\right) .
$$

Therefore, we get

$$
\bigcap_{s_{1}, s_{2} \in S}\left(B_{s_{1}, s_{2}} \cap A\right)=\bigcap_{x_{1}, x_{2} \in X}\left(B_{x_{1}, x_{2}} \cap A\right) .
$$

Let $N^{\prime}=\bigcap_{s_{1}, s_{2} \in S}\left(B_{s_{1}, s_{2}} \cap A\right)$. Then $\mu\left(N^{\prime}\right)=1$, which implies that for all $\omega \in N^{\prime}, T(\omega, x)$ are deterministic continuous operators. Therefore, $T$ has a unique random fixed point in $X$. Next, we show $\chi(\omega)$ is random and measurable. We construct a sequence of random variable $x_{n}(\omega)$. Let $x_{0}(\omega)$ be an arbitrary random variable and $x_{1}(\omega)=T\left(\omega, x_{0}(\omega)\right)$. Thus $x_{1}(\omega)$ is a random variable. Next, we get $x_{n+1}(\omega)=T\left(\omega, x_{n}(\omega)\right)$. By repeated generating, it gives that $\left\{x_{n}(\omega)\right\}_{n=1,2, \ldots}$ is a random variables sequence converging to $x(\omega)$. So, $x(\omega)$ is a random variable and therefore $x(\omega)$ is measurable. Thus $x(\omega)$ is a random fixed point of $\mathrm{T}$.

If we do not consider the simulation function, we obtain the corollary as follows.

Corollary 2.3. Assume that $(\Omega, \beta, \mu)$ is a complete probability measure space and $\mathrm{T}$ is an operator satisfying

$$
\left\|\mathrm{T}\left(\omega, \mathrm{x}_{1}(\omega)\right)-\mathrm{T}\left(\omega, \mathrm{x}_{2}(\omega)\right)\right\|<\left\|\mathrm{x}_{1}(\omega)-\mathrm{x}_{2}(\omega)\right\|
$$

almost surely for any $\mathrm{x}_{1}(\omega), \mathrm{x}_{2}(\omega) \in \mathrm{X}$, where $\mathrm{X}$ is a separable Banach space. Then a random fixed point of $\mathrm{T}$ exists in $\mathrm{X}$.

Proof. Suppose

$$
A=\{\omega \in \Omega: T(\omega, x) \text { is a continuous of } x\}
$$

and

$$
\mathrm{B}_{\mathrm{x}_{1}, \mathrm{x}_{2}}=\left\{\omega \in \Omega:\left\|\mathrm{T}\left(\omega, \mathrm{x}_{1}(\omega)\right)-\mathrm{T}\left(\omega, \mathrm{x}_{2}(\omega)\right)\right\|<\left\|\mathrm{x}_{1}(\omega)-\mathrm{x}_{2}(\omega)\right\|\right\} .
$$

Suppose $S$ is a countable dense set, $S \subset X$. Now, we prove that

$$
\bigcap_{x_{1}, x_{2} \in X}\left(B_{x_{1}, x_{2}} \cap A\right)=\bigcap_{s_{1}, s_{2} \in S}\left(B_{s_{1}, s_{2}} \cap A\right)
$$

Then for all $s_{1}, s_{2} \in S$, we get

$$
\left\|\mathrm{T}\left(\omega, \mathrm{s}_{1}(\omega)\right)-\mathrm{T}\left(\omega, \mathrm{s}_{2}(\omega)\right)\right\|<\left\|s_{1}(\omega)-\mathrm{s}_{2}(\omega)\right\| .
$$

Following the proof in Theorem 2.2, we get the result. 
From Theorem 2.2 and Corollary 2.3, we can illustrate example as follows.

Example 2.4. Assume that $X=\mathbb{R}$ with the usual norm of reals and $\Omega=\mathbb{R}$. Let $\beta$ be a $\sigma$-algebra of Lebesgue measurable sets of $\mathbb{R}$.

Now, we define the random operator $\mathrm{T}: \Omega \times \mathrm{X} \rightarrow \mathrm{X}$ by $\mathrm{T}(\omega, \mathrm{x})=\frac{\mathrm{x}}{2}$.

Since conditions of Theorem 2.2 and Corollary 2.3 are satisfied, we get that $x: \Omega \rightarrow X$ with $x(\omega)=0$ is the random fixed point of $T$ in $\mathbb{R}$.

We show the random fixed point of operator T by Figure 1.

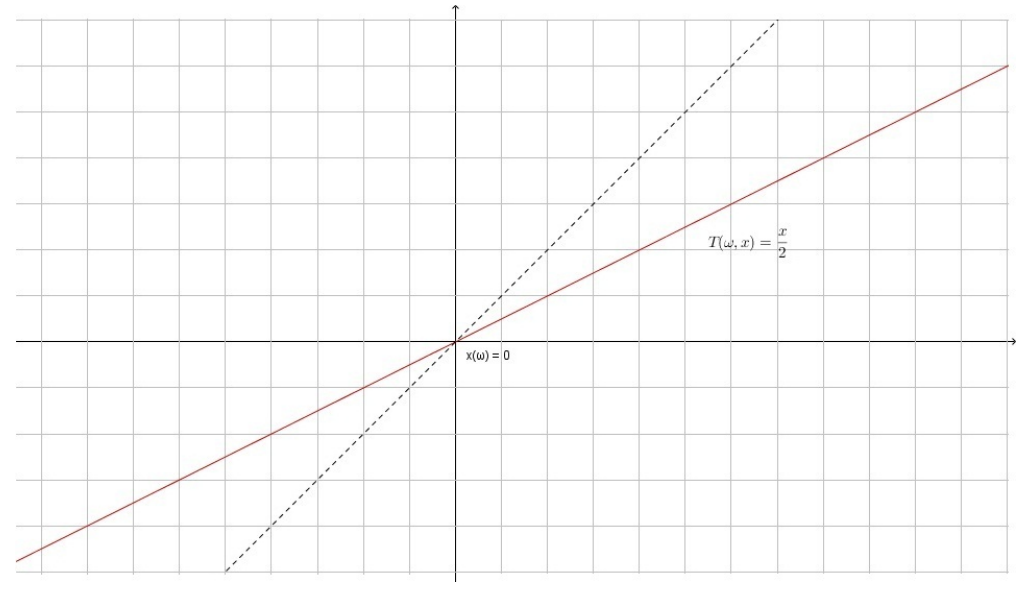

Figure 1: A random fixed point of operator $T(\omega, x)=\frac{\chi}{2}$ is $x(\omega)=0$.

\section{Applications to non-linear stochastic integral equations}

Now, we use Theorem 2.2 to show a solution of a non-linear stochastic integral equation exists in a Banach space. Assume that $S$ is a locally compact metric space and $(\Omega, \beta, \mu)$ is the probability measure space with $\beta$ being $\sigma$-algebra and $\mu$ the probability measure. We can write this equation of the Hammerstein type ([20]) as follows:

$$
x\left(t_{1} ; \omega\right)=h\left(t_{1} ; \omega\right)+\int_{S} k\left(t_{1} ; t_{2} ; \omega\right) f\left(t_{2} ; x\left(t_{2} ; \omega\right)\right) d \mu\left(t_{2}\right),
$$

where

(a) d is a metric imposed on product Cartesian of $S$;

(b) $\mu_{0}$ is a complete $\sigma$-finite measure imposed on the collection of Borel subsets of $S$;

(c) $\omega \in \Omega$ where $\Omega$ is the supporting set of $(\Omega, \beta, \mu)$;

(d) $x\left(t_{1} ; w\right)$ is the unknown vector-valued random variable for any $t_{1} \in S$;

(e) $h\left(t_{1} ; w\right)$ is the stochastic free term imposed for $t_{1} \in S$;

(f) $k\left(t_{1}, t_{2} ; w\right)$ is the stochastic kernel imposed for $t_{1}, t_{2} \in S$;

(g) $f\left(t_{1}, x\right)$ is a vector-valued function for $t_{1} \in S$ and $x$.

Note that (3.1) is called a Bochner integral (see in [29]).

Next, we suppose that the union of a countable family $\left\{C_{n}\right\}$ of compact sets by $C_{n+1} \subset C_{n}$ is imposed as $S$ so that, for each another compact set in $S$, there is $C_{i}$ which contains it (see [2]). 
We impose a space of all continuous functions from $S$ into $L_{2}(\Omega, \beta, \mu)$ by $C=C\left(S, L_{2}(\Omega, \beta, \mu)\right)$ by the topology of uniform convergence on compact sets of $S$, that is, $x\left(t_{1} ; \omega\right)$ is a vector-valued random variable for any fixed $t_{1} \in S$ so that

$$
\left\|x\left(t_{1} ; \omega\right)\right\|_{\mathrm{L}_{2}(\Omega, \beta, \mu)}^{2}=\int_{\Omega}\left|x\left(t_{1} ; \omega\right)\right|^{2} \mathrm{~d} \mu(\omega)<\infty .
$$

Observe that $C\left(S, L_{2}(\Omega, \beta, \mu)\right)$ is a locally convex space ([29]) whose topology is given by

$$
\left\|x\left(t_{1} ; \omega\right)\right\|_{n}=\sup _{t_{1} \in C_{n}}\left\|x\left(t_{1} ; \omega\right)\right\|_{L_{2}(\Omega, \beta, \mu)}
$$

which is the countable family of semi-norms for any $n \geqslant 1$. Moreover, because $L_{2}(\Omega, \beta, \mu)$ is complete, then $C\left(S, L_{2}(\Omega, \beta, \mu)\right)$ is complete relative to (3.2).

Later, we impose a Banach space of all bounded continuous functions from $S$ into $L_{2}(\Omega, \beta, \mu)$ by $B C=B C\left(S, L_{2}(\Omega, \beta, \mu)\right)$ by the norm

$$
\left\|x\left(t_{1} ; \omega\right)\right\|_{B C}=\sup _{t_{1} \in S}\left\|x\left(t_{1} ; \omega\right)\right\|_{L_{2}(\Omega, \beta, \mu)} .
$$

$B C \subset C$ is a space of all second order vector-valued stochastic processes imposed on $S$ which are bounded and continuous in mean square.

Now, we consider the functions $h\left(t_{1} ; \omega\right)$ and $f\left(t_{1}, x\left(t_{1} ; \omega\right)\right)$ to be in $C\left(S, L_{2}(\Omega, \beta, \mu)\right)$ space by respect to the stochastic kernel and suppose that, for any pair $\left(t_{1}, t_{2}\right), k\left(t_{1}, t_{2} ; \omega\right) \in L_{\infty}(\Omega, \beta, \mu)$ and the norm denoted by

$$
\left\|\left|k\left(t_{1}, t_{2} ; \omega\right)\right|\right\|=\left\|k\left(t_{1}, t_{2} ; \omega\right)\right\|_{L_{\infty}(\Omega, \beta, \mu)}=\mu-\operatorname{ess} \sup _{\omega \in \Omega}\left|k\left(t_{1}, t_{2} ; \omega\right)\right| .
$$

Also, we assume that $k\left(t_{1}, t_{2} ; \omega\right) \in L_{\infty}(\Omega, \beta, \mu)$ is so that $\left\|\left|k\left(t_{1}, t_{2} ; \omega\right)\right|\right\|=\left\|x\left(t_{2} ; \omega\right)\right\|_{L_{2}(\Omega, \beta, \mu)}$ is $\mu$ integrable by respect to $t_{2}$ for any $t_{1} \in S$ and $x\left(t_{2} ; \omega\right) \in C\left(S, L_{2}(\Omega, \beta, \mu)\right)$ and there is a real-valued function $G \mu$-a.e. on $S$ so that $G(S)\left\|x\left(t_{2} ; \omega\right)\right\|_{\left.L_{2}(\Omega, \beta, \mu)\right)}$ is $\mu$-integrable and, for any $\left(t_{1}, t_{2}\right)$ in $S \times S$,

$$
\left\|\left|k\left(t_{1}, u ; \omega\right)-k\left(t_{2}, u ; \omega\right)\right|\right\| \cdot\|x(u ; w)\|_{L_{2}(\Omega, \beta, \mu)} \leqslant G(u)\|x(u ; \omega)\|_{L_{2}(\Omega, \beta, \mu)} \quad \mu \text {-a.e.. }
$$

Later, suppose that, for almost every $t_{2} \in S, k\left(t_{1}, t_{2} ; \omega\right)$ is continuous in $t_{1}$ from $S$ into $L_{\infty}(\Omega, \beta, \mu)$.

Now, we impose the random integral operator $T$ on $C\left(S, L_{2}(\Omega, \beta, \mu)\right)$ by

$$
(T x)\left(t_{1} ; \omega\right)=\int_{S} k\left(t_{1}, t_{2} ; \omega\right) x\left(t_{2} ; \omega\right) d \mu\left(t_{2}\right),
$$

which is called a Bochner integral. By the assumptions on $k\left(t_{1}, t_{2} ; \omega\right)$, it follows that, for each $t_{1} \in S$, $(T x)\left(t_{1} ; \omega\right) \in L_{2}(\Omega, \beta, \mu)$ and $(T x)\left(t_{1} ; \omega\right)$ is continuous in mean square by Lebesgue's dominated convergence theorem, that is, $(T x)\left(t_{1} ; \omega\right) \in C\left(S, L_{2}(\Omega, \beta, \mu)\right)$.

Lemma 3.1 ([20]). The linear operator $T$ defined by (3.3) is continuous from $C\left(S, L_{2}(\Omega, \beta, \mu)\right)$ into itself.

Definition $3.2([1,18])$. Let B and D be Banach spaces. The pair (B, D) is called admissible by respect to a linear operator $\mathrm{T}$ if $\mathrm{T}(\mathrm{B}) \subset \mathrm{D}$.

Lemma 3.3 ([20]). If $T$ is a continuous linear operator from $C\left(S, L_{2}(\Omega, \beta, \mu)\right)$ into itself, $B, D \subset C\left(S, L_{2}(\Omega, \beta, \mu)\right)$ are Banach spaces stronger than $\mathrm{C}\left(\mathrm{S}, \mathrm{L}_{2}(\Omega, \beta, \mu)\right)$ so that $(\mathrm{B}, \mathrm{D})$ is admissible by respect to $\mathrm{T}$, then $\mathrm{T}$ is continuous from B into D.

By a random solution of (3.1), we mean a function

$$
x\left(t_{1} ; \omega\right) \in C\left(S, L_{2}(\Omega, \beta, \mu)\right)
$$

which satisfies (3.1) $\mu$-a.e.

By using Theorem 2.2, we are now in state to prove the theorem as follows. 
Theorem 3.4. Suppose that (3.1) is subject to the assumptions as follows:

(1) $\mathrm{B}$ and $\mathrm{D}$ are Banach spaces stronger than $\mathrm{C}\left(\mathrm{S}, \mathrm{L}_{2}(\Omega, \beta, \mu)\right)$ so that (B, D) is admissible by respect to the integral operator imposed by (3.3);

(2) $x\left(t_{1} ; \omega\right) \mapsto f\left(t_{1}, x\left(t_{1} ; \omega\right)\right)$ is an operator from $Q(\rho)=\left\{x\left(t_{1} ; \omega\right): x\left(t_{1} ; \omega\right) \in D,\left\|x\left(t_{1} ; \omega\right)\right\|_{D} \leqslant \rho\right\}$ into $B$ satisfying

$$
\zeta\left(\left\|f\left(t_{1}, x_{1}\left(t_{1}, w\right)\right)-f\left(t_{1}, x_{2}\left(t_{1}, w\right)\right)\right\|_{B},\left\|x_{1}\left(t_{1}, w\right)-x_{2}\left(t_{1}, w\right)\right\|\right) \geqslant 0
$$

for any $\mathrm{x}_{1}\left(\mathrm{t}_{1}, \omega\right), \mathrm{x}_{2}\left(\mathrm{t}_{1}, \omega\right) \in \mathrm{Q}(\rho)$;

(3) $h\left(t_{1} ; \omega\right) \in D$,

then a unique stochastic solution of (3.1) exists in $\mathrm{Q}(\rho)$ provided that

$$
\left\|h\left(t_{1}, \omega\right)\right\|_{D}+\sigma(\omega)\left\|f\left(t_{1}, 0\right)\right\|_{B} \leqslant \rho(1-\sigma(\omega)),
$$

where the norm of $\mathrm{T}(\omega)$ is denoted by $\sigma(\omega)$.

Proof. Let a mapping $U(\omega): \mathrm{Q}(\rho) \rightarrow \mathrm{D}$ defined by

$$
(\mathcal{U} x)\left(t_{1}, \omega\right)=h\left(t_{1}, \omega\right)+\int_{S} k\left(t_{1}, t_{2}, \omega\right) f\left(s, x\left(t_{2}, \omega\right)\right) d_{\mu_{0}}(s) .
$$

Then we get

$$
\begin{aligned}
\|\left(\mathcal{U}(x)\left(t_{1}, \omega\right) \|_{D}\right. & \leqslant\left\|h\left(t_{1}, \omega\right)\right\|_{D}+\sigma(\omega)\left\|f\left(t_{1}, x\left(t_{1}, \omega\right)\right)\right\|_{B} \\
& =\left\|h\left(t_{1}, \omega\right)\right\|_{D}+\sigma(\omega)\left\|f\left(t_{1}, 0\right)+f\left(t_{1}, x\left(t_{1}, \omega\right)\right)-f\left(t_{1}, 0\right)\right\|_{B} \\
& \leqslant\left\|h\left(t_{1}, \omega\right)\right\|_{D}+\sigma(\omega)\left\|f\left(t_{1}, 0\right)\right\|_{B}+\sigma(\omega)\left\|f\left(t_{1}, x\left(t_{1}, \omega\right)\right)-f\left(t_{1}, 0\right)\right\|_{B} .
\end{aligned}
$$

Thus, it follows by (3.4) that

$$
\zeta\left(\left\|f\left(t_{1}, x\left(t_{1}, \omega\right)\right)-f\left(t_{1}, 0\right)\right\|_{B},\left\|x\left(t_{1}, w\right)\right\|_{D}\right) \geqslant 0,
$$

which implies that

$$
\left\|f\left(t_{1}, x\left(t_{1}, w\right)\right)-f\left(t_{1}, 0\right)\right\|_{B}<\left\|x\left(t_{1}, w\right)\right\|_{D}
$$

Therefore, we obtain

$$
\left\|f\left(t_{1}, x\left(t_{1}, w\right)\right)-f\left(t_{1}, 0\right)\right\|_{B} \leqslant \rho .
$$

Therefore, by (3.5), we have

$$
\begin{aligned}
\left\|(\mathcal{U} x)\left(t_{1}, \omega\right)\right\|_{D} & \leqslant\left\|h\left(t_{1}, \omega\right)\right\|_{D}+\sigma(\omega)\left\|f\left(t_{1}, 0\right)\right\|_{B}+\sigma(\omega)\left\|f\left(t_{1}, x\left(t_{1}, \omega\right)\right)-f\left(t_{1}, 0\right)\right\|_{B} \\
& \leqslant\left\|h\left(t_{1}, \omega\right)\right\|_{D}+\sigma(\omega)\left\|f\left(t_{1}, 0\right)\right\|_{B}+\sigma(\omega) \rho<\rho
\end{aligned}
$$

and so, by (3.6), $(\mathcal{U} x)\left(t_{1}, \omega\right) \in Q(\rho)$. Thus, for any $x_{1}\left(t_{1}, w\right), x_{2}\left(t_{1}, \omega\right) \in Q(\rho)$ and, by $(2)$, we get

$$
\begin{aligned}
\left\|\left(U x_{1}\right)\left(t_{1}, w\right)-\left(U x_{2}\right)\left(t_{1}, w\right)\right\|_{D} & =\left\|\int_{S} k\left(t_{1}, t_{2}, w\right)\left[f\left(t_{2}, x_{1}\left(t_{2}, w\right)\right)-f\left(t_{2}, x_{2}\left(t_{2}, w\right)\right)\right] d \mu_{0}(s)\right\|_{D} \\
& \leqslant \sigma(\omega)\left\|f\left(t_{2}, x_{1}\left(t_{2}, \omega\right)\right)-f\left(t_{2}, x_{2}\left(t_{2}, w\right)\right)\right\|_{B} \leqslant\left\|x_{1}\left(t_{1}, w\right)-x_{2}\left(t_{1}, \omega\right)\right\|_{D} .
\end{aligned}
$$

Consequently, $\mathcal{U}(\omega)$ is a random contraction mapping over $Q(\rho)$. Therefore, by Theorem 2.2, there is a unique $x^{*}\left(t_{1}, \omega\right) \in Q(\rho)$, which is a random fixed point of $\mathcal{U}$, i.e., $x^{*}$ is a stochastic solution of equation (3.1). This completes the proof. 
Example 3.5. Consider the non-linear stochastic integral equation as follows:

$$
x\left(t_{1} ; \omega\right)=\int_{0}^{\infty} \frac{e^{-t_{1}-t_{2}}}{8\left(1+\left|x\left(t_{2} ; \omega\right)\right|\right)} d t_{2} .
$$

Next, we compare between equations (3.1) and (3.5), we get that $h\left(t_{1} ; \omega\right)=0, k\left(t_{1} ; t_{2} ; \omega\right)=\frac{1}{2} e^{-t_{1}-t_{2}}$ and $f\left(t_{2} ; x\left(t_{2} ; \omega\right)\right)=\frac{1}{4\left(1+\left|x\left(t_{2} ; \omega\right)\right|\right)}$. Then, equation (3.4) holds.

Also, comparing with integral equation (3.3), we get that $\sigma(\omega)=\frac{1}{2}$ which $\sigma(\omega)$ is the norm of $T(\omega)$. Thus, all assumptions of Theorem 3.4 are satisfied and therefore, random operator $\mathrm{T}$ has a random fixed point.

\section{Acknowledgment}

The first author was supported by Rajamangala University of Technology Lanna (RMUTL) for Ph.D. program at King Mongkut's University of Technology Thonburi (KMUTT). The second author was supported by the Theoretical and Computational Science (TaCS) Center (Project Grant No. Tacs2559-2).

\section{References}

[1] J. Achari, On a pair of random generalized nonlinear contractions, Internat. J. Math. Math. Sci., 6 (1983), 467-475. 1, 3.2

[2] R. F. Arens, A topology for spaces of transformations, Ann. of Math., 47 (1946), 480-495. 3

[3] S. Banach, Sur les opérations dans les ensembles abstraits et leur application aux équations intégrales, Fund. Math., 3 (1922), 133-181. 1

[4] I. Beg, N. Shahzad, Random fixed points of random multivalued operators on Polish spaces, Nonlinear Anal., 20 (1993), 835-847. 1

[5] A. T. Bharucha-Reid, Fixed point theorems in probabilistic analysis, Bull. Amer. Math. Soc., 82 (1976), 641-657. 1

[6] Y. J. Cho, J. Li, N. J. Huang, Random Ishikawa iterative sequence with errors for approximating random fixed points, Taiwanese J. Math., 12 (2008), 51-61. 1

[7] O. Hanš, Reduzierende zufällige Transformationen, (German) Czechoslovak Math. J., 7 (1957), 154-158. 1

[8] O. Hanš, Random operator equations, Proc. 4th Berkeley Sympos. Math. Statist. and Prob., Univ. California Press, Berkeley, Calif., II (1961), 185-202. 1

[9] S. Itoh, Random fixed-point theorems with an application to random differential equations in Banach spaces, J. Math. Anal. Appl., 67 (1979), 261-273. 1

[10] J. S. Jung, Y. J. Cho, S. M. Kang, B. S. Lee, B. S. Thakur, Random fixed point theorems for a certain class of mappings in Banach spaces, Czechoslovak Math. J., 50 (2000), 379-396. 1

[11] F. Khojasteh, S. Shukla, S. Radenović, A new approach to the study of fixed point theory for simulation functions, Filomat, 29 (2015), 1188-1194. 1

[12] P. Kumam, Random common fixed points of single-valued and multivalued random operators in a uniformly convex Banach space, J. Comput. Anal. Appl., 13 (2011), 368-375. 1

[13] P. Kumam, W. Kumam, Random fixed points of multivalued random operators with property (D), Random Oper. Stoch. Equ., 15 (2007), 127-136.

[14] W. Kumam, P. Kumam, Random fixed point theorems for multivalued subsequentially limit-contractive maps satisfying inwardness conditions, J. Comput. Anal. Appl., 14 (2012), 239-251.

[15] P. Kumam, S. Plubtieng, Random coincidence and random common fixed points of nonlinear multivalued random operators, Thai J. Math., 5 (2007), 155-163.

[16] P. Kumam, S. Plubtieng, The characteristic of noncompact convexity and random fixed point theorem for set-valued operators, Czechoslovak Math. J., 57 (2007), 269-279.

[17] P. Kumam, S. Plubtieng, Some random fixed point theorems for random asymptotically regular operators, Demonstratio Math., 42 (2009), 131-141. 1

[18] A. C. H. Lee, W. J. Padgett, On random nonlinear contractions, Math. Systems Theory, 11 (1977/78), 77-84. 3.2

[19] A. Mukherjee, Transformation aleatoires separable theorem all point xed aleatoire, C. R. Acad. Sci. Paris, Ser. A-B, 263 (1966), 393-395. 1

[20] W. J. Padgett, On a nonlinear stochastic integral equation of the Hammerstein type, Proc. Amer. Math. Soc., 38 (1973), 625-631. 1, 3, 3.1, 3.3

[21] S. Plubtieng, P. Kumam, Random fixed point theorems for multivalued nonexpansive non-self-random operators, J. Appl. Math. Stoch. Anal., 2006 (2006), 9 pages. 1 
[22] E. Rothe, Zur Theorie der topologischen Ordnung und der Vektorfelder in Banachschen Räumen, (German) Compositio Math., 5 (1938), 177-197. 1

[23] M. Saha, On some random fixed points of mappings over a Banach space with a probability measure, Proc. Natl. Acad. Sci. India Sect. A Phys. Sci., 76 (2006), 219-224. 1

[24] M. Saha, L. Debnath, Random fixed point of mappings over a Hilbert space with a probability measure, Adv. Stud. Contemp. Math. (Kyungshang), 18 (2009), 97-104.

[25] M. Saha, D. Dey, Some random fixed point theorems for ( $\theta$, L)-weak contractions, Hacet. J. Math. Stat., 41 (2012), $795-$ 812.

[26] M. Saha, A. Ganguly, Random fixed point theorem on a iri-type contractive mapping and its consequence, Fixed Point Theory Appl., 2012 (2012), 18 pages. 1, 2

[27] V. M. Sehgal, C. Waters, Some random fixed point theorems for condensing operators, Proc. Amer. Math. Soc., 90 (1984), 425-429. 1

[28] A. Špaček, Zufüllige Gleichungen, (German) Czechoslovak Math. J., 5 (1955), 462-466. 1

[29] K. Yosida, Functional analysis, Die Grundlehren der Mathematischen Wissenschaften, Band 123 Academic Press, Inc., New York; Springer-Verlag, Berlin, (1965). 3 